\title{
The pointed intrinsic flat distance between locally integral current spaces
}

\author{
Shu Takeuchi *
}

September 21, 2018

\begin{abstract}
In this note we define a distance between two pointed locally integral current spaces. We prove that a sequence of pointed locally integral current spaces converges with respect to this distance if and only if it converges in the sense of Lang-Wenger. This enables us to state the compactness theorem by Lang-Wenger for pointed locally integral current spaces in terms of a distance function.
\end{abstract}

\section{Contents}

1 Introduction 1

2 Locally integral currents in metric spaces 3

3 The pointed intrinsic flat distance $\quad 7$

\section{Introduction}

Ambrosio and Kirchheim defined currents in metric spaces in [AK00]. We begin with a rough review of currents in metric spaces, see the next section for details. Let $X$ be a complete separable metric space, $k$ be a nonnegative integer and $\mathcal{D}_{\mathrm{AK}}^{k}(X):=\operatorname{Lip}_{\mathrm{b}}(X) \times$ $(\operatorname{Lip}(X))^{k}$. We call a multilinear function $T: \mathcal{D}_{\mathrm{AK}}^{k}(X) \rightarrow \mathbb{R}$ a $k$-dimensional current in $X$ if $T$ possesses continuity, locality (see Definition 2.1) and finiteness of the mass. "Finiteness of the mass" means that there exists a finite Borel measure $\mu$ on $X$ such that

$$
\left|T\left(f, \pi_{1}, \ldots, \pi_{k}\right)\right| \leq \prod_{i=1}^{k} \operatorname{Lip}\left(\pi_{i}\right) \int_{X}|f| d \mu
$$

holds for all $\left(f, \pi_{1}, \ldots, \pi_{k}\right) \in \mathcal{D}_{\mathrm{AK}}^{k}(X) .\|T\|$ stands for the minimal measure $\mu$ satisfying (1.1), and we define the mass of $T$ by $\mathbf{M}(T):=\|T\|(X)$. Let $T_{1}$ and $T_{2}$ be $k$-dimensional integral currents in $X$. We define the flat distance between $T_{1}$ and $T_{2}$ in $X$ by

$$
\mathcal{F}^{X}\left(T_{1}, T_{2}\right):=\inf _{U, V}(\mathbf{M}(U)+\mathbf{M}(V)),
$$

where the infimum is taken over all $U \in \mathbf{I}_{k}(X)$ and $V \in \mathbf{I}_{k+1}(X)$ with $T_{1}-T_{2}=U+\partial V$ and $\mathbf{I}_{k}(X)$ denotes the set of all $k$-dimensional integral currents in $X$.

${ }^{*}$ Tohoku University, shu.takeuchi.q8@dc.tohoku.ac.jp 
In order to discuss integral currents in different metric spaces, Sormani and Wenger defined the intrinsic flat distance in [SW11]. Roughly speaking, the intrinsic flat distance between two $k$-dimensional integral current spaces $\left(X_{1}, d_{1}, T_{1}\right)$ and $\left(X_{2}, d_{2}, T_{2}\right)$ is defined by

$$
d_{\mathcal{F}}\left(\left(X_{1}, d_{1}, T_{1}\right),\left(X_{2}, d_{2}, T_{2}\right)\right):=\inf \mathcal{F}^{Z}\left(\varphi_{1 \#} T_{1}, \varphi_{2 \#} T_{2}\right)
$$

where the infimum is taken over all complete metric spaces $(Z, d)$ and isometric embeddings $\varphi_{1}:\left(X_{1}, d_{1}\right) \hookrightarrow(Z, d)$ and $\varphi_{2}:\left(X_{2}, d_{2}\right) \hookrightarrow(Z, d)$. Furthermore, they proved a compactness theorem with respect to this distance. The statement of the theorem is as follows: fix a sequence of complete separable metric spaces $\left\{\left(X_{n}, d_{n}\right)\right\}_{n}$ which are uniformly bounded and uniformly totally bounded. Let $k \geq 1$ and $T_{n} \in \mathbf{I}_{k}\left(X_{n}\right)$. If we assume $\sup _{n}\left(\mathbf{M}\left(T_{n}\right)+\mathbf{M}\left(\partial T_{n}\right)\right)<\infty$, then there exist an integral current space $(X, d, T)$ and a subsequence $n(j)$ such that $\left(X_{n(j)}, d_{n(j)}, T_{n(j)}\right)$ converges to $(X, d, T)$ with respect to the intrinsic flat distance. Note that $(X, d)$ is not necessarily isometric to the Gromov-Hausdorff limit space of $\left(X_{n(j)}, d_{n(j)}\right)$ (see Figure 2 in [SW11]). Also note that even if $\left(X_{n}, d_{n}, T_{n}\right)$ converges to $(X, d, T)$ with respect to $d_{\mathcal{F}}$, the condition $\sup _{n}\left(\mathbf{M}\left(T_{n}\right)+\mathbf{M}\left(\partial T_{n}\right)\right)<\infty$ does not always hold. It is not difficult to construct a sequence $\left\{T_{n}\right\}_{n} \subset \mathbf{I}_{k}\left(\mathbb{R}^{k+1}\right)$ which converges with respect to flat distance and satisfies $\sup _{n} \mathbf{M}\left(T_{n}\right)=\infty$.

Now, let us discuss the case when the current spaces are pointed locally integral current spaces, which may have infinite mass. Let $X$ be a complete separable metric space, $x \in X$, $k \geq 1$ and $T \in \mathbf{I}_{\mathrm{Loc}, k}(X)$, where $\mathbf{I}_{\mathrm{Loc}, k}(X)$ denotes the set of all $k$-dimensional locally integral currents in $X$. We call $(X, x, T)$ a $k$-dimensional pointed locally integral current space, and $\mathcal{M}_{*}^{k}$ denotes the space of $k$-dimensional pointed locally integral current spaces. In [LW11], Lang and Wenger studied the following convergence in $\mathcal{M}_{*}^{k}$ : for a sequence $\left\{\left(X_{n}, x_{n}, T_{n}\right)\right\}_{n} \subset \mathcal{M}_{*}^{k}$, we say that $\left(X_{n}, x_{n}, T_{n}\right)$ converges to $(Z, z, T) \in \mathcal{M}_{*}^{k}$ if there is an isometric embedding $\varphi_{n}: X_{n} \hookrightarrow Z$ such that $d_{Z}\left(\varphi_{n}\left(x_{n}\right), z\right) \rightarrow 0(n \rightarrow \infty)$ and $\varphi_{n \#} T_{n}$ converges to $T$ in the local flat topology (Definition 2.14). Then they proved a compactness theorem for pointed locally integral current spaces with respect to this convergence. It is a natural question that this convergence can be written in terms of a distance function as in [SW11], that is, this convergence is metrizable or not. By using the same idea as the intrinsic flat distance in [SW11] and the pointed Gromov-Hausdorff distance in [Gro81], we define the pointed intrinsic flat distance $d_{\mathcal{F} *}$ in $\mathcal{M}_{*}^{k}$, whose convergence is compatible with the above convergence (Definition 3.1, Proposition 3.4 and Proposition 3.6): consequently, by the compactness theorem (Theorem 1.1 in [LW11]: see [LW11], [Wen11], [Wen05] and [Wen07] for the proof), we obtain the following theorem:

Theorem 1.1. Let $k \geq 1$. Assume that a sequence of $k$-dimensional pointed locally integral current spaces $\left\{\left(X_{n}, x_{n}, T_{n}\right)\right\}_{n} \subset \mathcal{M}_{*}^{k}$ satisfies

$$
\sup _{n}\left(\left\|T_{n}\right\|+\left\|\partial T_{n}\right\|\right)\left(\bar{B}_{r}\left(x_{n}\right)\right)<\infty
$$

for all $r>0$, where $\bar{B}_{r}\left(x_{n}\right)$ is the closed ball of radius $r$ centered at $x_{n}$. Then there exist a subsequence $\left\{\left(X_{n(j)}, x_{n(j)}, T_{n(j)}\right)\right\}_{j}$ and $(Z, z, T) \in \mathcal{M}_{*}^{k}$ such that

$$
d_{\mathcal{F} *}\left(\left(X_{n(j)}, x_{n(j)}, T_{n(j)}\right),(Z, z, T)\right) \rightarrow 0
$$

as $j \rightarrow \infty$.

In particular, we see that the convergence defined in [LW11] for pointed locally integral current spaces is "intrinsic." Finally, we mention the $L_{\text {loc }}^{2}$-convergence of orientations of 
Riemannian manifolds. Let $\left(X, x, \mathcal{H}^{k}\right)$ be a Ricci limit space and $\left\{\left(X_{n}^{k}, x_{n}, \mathcal{H}^{k}\right)\right\}_{n}$ be a sequence of $k$-dimensional Riemannian manifolds with $\operatorname{Ric}_{X_{n}} \geq-(k-1)$ and $\mathcal{H}^{k}\left(B_{1}\left(x_{n}\right)\right) \geq$ $v>0$. Let $\omega_{n}$ (resp. $\left.\omega\right)$ be an orientation of $X_{n}$ (resp. $\left.X\right)$. Then we see that $\left(X_{n}, x_{n}, \mathcal{H}^{k}\right)$ mGH-converges to $\left(X, x, \mathcal{H}^{k}\right)$ and $\omega_{n} L_{\text {loc }}^{2}$-converges to $\omega$ simultaneously if and only if $X_{n}$ converges to $X$ as pointed locally integral current spaces with respect to $d_{\mathcal{F} *}$ (see [Hon17] for details).

\section{Locally integral currents in metric spaces}

In this section, we recall the definition of $k$-dimensional locally integral currents and related notions. See [LW11] for details.

Throughout this section, let $X$ be a complete separable metric space. We define classes of Lipschitz functions as follows:

$\operatorname{Lip}_{\text {Loc }}(X):=\left\{f: X \rightarrow \mathbb{R} ;\left.f\right|_{B}\right.$ is Lipschitz for any bounded set $\left.B \subset X\right\}$,

$\operatorname{Lip}(X):=\{f: X \rightarrow \mathbb{R} ; f$ is Lipschitz $\}$,

$\operatorname{Lip}_{1}(X):=\{f \in \operatorname{Lip}(X) ;|f(x)-f(y)| \leq d(x, y)$ for all $x, y \in X\}$,

$\operatorname{Lip}_{\mathrm{b}}(X):=\{f \in \operatorname{Lip}(X) ; f$ is bounded $\}$,

$\operatorname{Lip}_{\mathrm{B}}(X):=\left\{f \in \operatorname{Lip}_{\mathrm{b}}(X) ; \operatorname{spt} f\right.$ is bounded $\}$,

where spt $f:=\overline{\{x \in X ; f(x) \neq 0\}}$. More generally, for a metric space $X^{\prime}$, we define

$\operatorname{Lip}_{\text {Loc }}\left(X, X^{\prime}\right):=\left\{f: X \rightarrow X^{\prime} ;\left.f\right|_{B}\right.$ is Lipschitz for any bounded set $\left.B \subset X\right\}$, $\operatorname{Lip}\left(X, X^{\prime}\right):=\left\{f: X \rightarrow X^{\prime} ; f\right.$ is Lipschitz $\}$.

$\operatorname{Lip}(f)$ denotes the Lipschitz constant of $f \in \operatorname{Lip}(X)$, that is, $\operatorname{Lip}(f):=\sup \{\mid f(x)-$ $f(y) \mid / d(x, y) ; x, y \in X, x \neq y\}$. For $r>0, B_{r}(x)$ (resp. $\left.\bar{B}_{r}(x)\right)$ denotes the open (resp. closed) ball centered at $x \in X$ with radius $r$. Similarly, for a subset $A \subset X, B_{r}(A)$ (resp. $\bar{B}_{r}(A)$ ) denotes the open (resp. closed) $r$-neighborhood of $A$.

For $k \geq 0$, let $\mathcal{D}^{k}(X):=\operatorname{Lip}_{\mathrm{B}}(X) \times\left(\operatorname{Lip}_{\mathrm{Loc}}(X)\right)^{k}$. If $X^{n}$ is an $n$-dimensional Riemannian manifold, by Rademacher's theorem, any element $\left(f, \pi_{1}, \ldots, \pi_{k}\right) \in \mathcal{D}^{k}(X)$ determines a $k$-dimensional differential form $f d \pi_{1} \wedge \cdots \wedge d \pi_{k} \mathcal{H}^{n}$-a.e. on $X$. Thus we write $\left(f, \pi_{1}, \ldots, \pi_{k}\right)$ as $f d \pi_{1} \wedge \cdots \wedge d \pi_{k}$ or $f d \pi$ for short.

Definition 2.1. A function $T: \mathcal{D}^{k}(X) \rightarrow \mathbb{R}$ is called a $k$-dimensional metric functional on $X$ if the following properties hold:

(i) (multilinearity) $T$ is multilinear.

(ii)(continuity) $\lim _{j \rightarrow \infty} T\left(f d \pi^{j}\right)=T(f d \pi)$ holds whenever $\pi_{i}^{j}$ pointwisely converges to $\pi_{i}$ for any $i=1, \ldots, k$ with $\sup _{j} \operatorname{Lip}\left(\left.\pi_{i}^{j}\right|_{B}\right)<\infty$ for any bounded set $B \subset X$.

(iii)(locality) If $\pi_{i}$ is constant on $\bar{B}_{\delta}(\operatorname{spt} f)$ for some $\delta>0$, then $T(f d \pi)=0$.

A typical example is as follows:

Definition 2.2. Let $\theta \in L_{\text {loc }}^{1}\left(\mathbb{R}^{k}\right)$, then a function $[\theta]: \mathcal{D}^{k}\left(\mathbb{R}^{k}\right) \rightarrow \mathbb{R}$ defined by

$$
[\theta]\left(f d \pi_{1} \wedge \cdots \wedge d \pi_{k}\right):=\int_{\mathbb{R}^{k}} f \theta \operatorname{det}(\nabla \pi) d \mathcal{L}^{k}
$$

is a $k$-dimensional metric functional on $\mathbb{R}^{k}$, where $\mathcal{L}^{k}$ denotes the Lebesgue measure on $\mathbb{R}^{k}$. 
Now we define the pushforward, the restriction and the boundary of a metric functional.

Definition 2.3. Let $T$ be a $k$-dimensional metric functional on $X$.

(i) Let $\varphi \in \operatorname{Lip}_{\text {Loc }}\left(X, X^{\prime}\right)$, and assume that for any bounded set $A \subset X^{\prime} \varphi^{-1}(A)$ is also bounded. Then we define a $k$-dimensional metric functional $\varphi_{\#} T$ on $X^{\prime}$ by

$$
\left(\varphi_{\#} T\right)\left(f, \pi_{1}, \ldots, \pi_{k}\right):=T\left(f \circ \varphi, \pi_{1} \circ \varphi, \ldots, \pi_{k} \circ \varphi\right) .
$$

$\varphi_{\#} T$ is called the pushforward of $T$ with respect to $\varphi$.

(ii) Let $l \in[0, k]$ be an integer and $g d \tau \in\left(\operatorname{Lip}_{\text {Loc }}(X)\right)^{l+1}$. We define a $(k-l)$-dimensional metric functional $T\llcorner(g d \tau)$ on $X$ by

$$
\left(T\llcorner(g d \tau))(f d \pi):=T\left(f g, \tau_{1}, \ldots, \tau_{l}, \pi_{1}, \ldots, \pi_{k-l}\right) .\right.
$$

$T\llcorner(g d \tau)$ is called the restriction of $T$ to $g d \tau$.

(iii) Let $k \geq 1$. We define a $(k-1)$-dimentional metric functional $\partial T$ by

$$
(\partial T)(f d \pi):=T\left(1 d f \wedge d \pi_{1} \wedge \cdots \wedge d \pi_{k-1}\right) .
$$

$\partial T$ is called the boundary of $T$.

Now we define the mass of a $k$-dimensional metric functional $T$.

Definition 2.4. Let $T$ be a $k$-dimensional metric functional on $X$. For any open set $O \subset X$, define

$$
\|T\|(O):=\sup \sum_{j=1}^{N} T\left(f^{j} d \pi_{1}^{j} \wedge \cdots \wedge d \pi_{k}^{j}\right)
$$

where the supremum is taken over all $N \in \mathbb{N}$ and $f^{j} d \pi_{1}^{j} \wedge \cdots \wedge d \pi_{k}^{j} \in \operatorname{Lip}_{\mathrm{B}}(X) \times\left(\operatorname{Lip}_{1}(X)\right)^{k}$ with spt $f^{j} \subset O$ and $\sum_{j=1}^{N}\left|f^{j}\right| \leq 1$. For all $A \subset X$, put

$$
\|T\|(A):=\inf \{\|T\|(O) ; O \supset A \text { is open }\} .
$$

It is natural to ask whether $\|T\|: 2^{X} \rightarrow \mathbb{R}_{\geq 0} \cup\{\infty\}$ is an outer measure or not. The following lemma gives an answer to this question. See Proposition 2.2 in [LW11] for the proof.

Lemma 2.5. Let $T$ be a $k$-dimensional metric functional on $X$. Assume that for any bounded open set $O \subset X$ and $\epsilon>0$, there exists a compact set $K \subset O$ such that $\|T\|(O \backslash K)<\epsilon$. Then $\|T\|$ is an outer measure satisfying that

(i) any Borel set is $\|T\|$-measurable,

(ii) for any $A \in 2^{X}$, there exists a Borel set $B$ such that $B \supset A$ and $\|T\|(A)=\|T\|(B)$,

(iii) there exists a $\sigma$-compact set $\Sigma \subset X$ such that $\|T\|\left(\Sigma^{c}\right)=0$.

For a metric functional $T$ satisfying the assumption of Lemma 2.5, let

$$
\operatorname{spt} T:=\left\{x \in X ;\|T\|\left(B_{r}(x)\right)>0 \text { for all } r>0\right\} .
$$

Using Definition 2.4 and Lemma 2.5, we define metric currents with locally finite mass. Note that it may satisfy $\|T\|(X)=\infty$, while [SW11] deals with metric currents with $\|T\|(X)<\infty$. 
Definition 2.6. We say that a $k$-dimensional metric functional $T$ is a metric current with locally finite mass if $\|T\|(O)<\infty$ holds for any bounded open set $O \subset X$ and the assumption of Lemma 2.5 holds. $\mathbf{M}_{\mathrm{Loc}, k}(X)$ denotes the vector space consisting of all $k$-dimensional metric currents with locally finite mass.

The next proposition will play a key role later, see Proposition 2.3 in [LW11] for the proof.

Proposition 2.7. Let $T \in \mathbf{M}_{\mathrm{Loc}, k}(X)$. Then

$$
|T(f d \pi)| \leq \prod_{i=1}^{k} \operatorname{Lip}\left(\left.\pi_{i}\right|_{\text {spt } f}\right) \int_{X}|f| d\|T\|
$$

holds for all $f d \pi \in \mathcal{D}^{k}(X)$.

In order to extend the domain of $T \in \mathbf{M}_{\mathrm{Loc}, k}(X)$, let us use the following notation:

$$
\begin{aligned}
& \mathscr{B}_{\mathrm{Loc}}^{\infty}(X):=\left\{f: X \rightarrow \mathbb{R} ; \text { Borel measurable, } \sup _{A}|f|<\infty \text { for any bounded set } A \subset X\right\}, \\
& \mathscr{B}^{\infty}(X):=\left\{f: X \rightarrow \mathbb{R} ; \text { Borel measurable, } \sup _{X}|f|<\infty\right\}, \\
& \mathscr{B}_{\mathrm{B}}^{\infty}(X):=\left\{f \in \mathscr{B}^{\infty}(X) ; \text { spt } f \text { is bounded }\right\} .
\end{aligned}
$$

In the following, we use the next lemma.

Lemma 2.8. Let $T \in \mathbf{M}_{\mathrm{Loc}, k}(X), f \in \mathscr{B}_{\mathrm{B}}^{\infty}(X)$ and $N$ be a bounded neighborhood of spt $f$. Then there exists $\left\{f_{n}\right\}_{n} \subset \operatorname{Lip}_{\mathrm{B}}(X)$ such that spt $f_{n} \subset N$ holds for all $n$ and $f_{n} \rightarrow f$ in $L^{1}(\|T\|)$.

Proof. Fix an arbitrary $n \in \mathbb{N}$. Let $R>0$ be a positive number such that $B_{R}(\operatorname{spt} f) \subset N$. By Definition 2.6, there exists a compact set $K \subset B_{R}($ spt $f)$ such that $\|T\|\left(B_{R}(\operatorname{spt} f) \backslash K\right)<$ $\left(n\left(1+\sup _{X}|f|\right)\right)^{-1}$. Then we have

$$
\int_{X}\left|f-f \chi_{K}\right| d\|T\|=\int_{B_{R}(\operatorname{spt} f) \backslash K}|f| d\|T\|<\frac{1}{n},
$$

where $\chi_{K}$ is the indicator function of $K$. Moreover, by the definition of the integral, there exist finite Borel sets $B_{1}, \ldots, B_{m}$ and real numbers $a_{1}, \ldots, a_{m}$ such that $B_{i} \subset K$ for all $i=1, \ldots, m$ and that

$$
\int_{X}\left|f \chi_{K}-\sum_{i=1}^{m} a_{i} \chi_{B_{i}}\right| d\|T\|<\frac{1}{n}
$$

Finally, for all $i$, there exists $f_{n, i} \in \operatorname{Lip}_{\mathrm{B}}(X)$ such that

$$
\int_{X}\left|a_{i} \chi_{B_{i}}-f_{n, i}\right| d\|T\|<\frac{1}{n m} .
$$

Indeed, for any $\epsilon>0$, define $f_{n, i, \epsilon} \in \operatorname{Lip}_{\mathrm{B}}(X)$ by $f_{n, i, \epsilon}(x):=a_{i} \max \left\{0,1-\epsilon^{-1} d\left(x, B_{i}\right)\right\}$. Then one can find such functions by letting $\epsilon \rightarrow 0$. Taking $f_{n}:=\sum_{i=1}^{m} f_{n, i}$ completes the proof. 
Definition 2.9. Let $T \in \mathbf{M}_{\mathrm{Loc}, k}(X)$. For $\left(f, \pi_{1}, \ldots, \pi_{k}\right) \in \mathscr{B}_{\mathrm{B}}^{\infty}(X) \times\left(\operatorname{Lip}_{\mathrm{Loc}}(X)\right)^{k}$, define

$$
T\left(f, \pi_{1}, \ldots, \pi_{k}\right):=\lim _{n \rightarrow \infty} T\left(f_{n} d \pi\right)
$$

where $N$ is a bounded neighborhood of spt $f$, and $\left\{f_{n}\right\}_{n} \subset \operatorname{Lip}_{\mathrm{B}}(X)$ satisfies spt $f_{n} \subset N$ and $f_{n} \rightarrow f$ in $L^{1}(\|T\|)$, as in Lemma 2.8 .

The limit in (2.2) exists since $\left\{T\left(f_{n} d \pi\right)\right\}_{n}$ is a Cauchy sequence by (2.1) and Lemma 2.8. If we take another bounded neighborhood $N^{\prime}$ of spt $f$ and another sequence $\left\{g_{n}\right\}_{n} \subset$ $\operatorname{Lip}_{\mathrm{B}}(X)$ with spt $g_{n} \subset N^{\prime}$ and $g_{n} \rightarrow f$ in $L^{1}(\|T\|)$, then

$$
\left|T\left(f_{n} d \pi\right)-T\left(g_{n} d \pi\right)\right| \leq \prod_{i=1}^{k} \operatorname{Lip}\left(\left.\pi_{i}\right|_{N \cup N^{\prime}}\right) \int_{X}\left|f_{n}-g_{n}\right| d\|T\| \rightarrow 0(n \rightarrow \infty)
$$

holds. Therefore the limit in (2.2) does not depend on the choice of $N$ and $\left\{f_{n}\right\}_{n}$.

Now we define the restriction to $g d \tau \in \mathscr{B}_{\mathrm{Loc}}^{\infty}(X) \times\left(\operatorname{Lip}_{\mathrm{Loc}}(X)\right)^{l}$ :

Definition 2.10. Let $T \in \mathbf{M}_{\mathrm{Loc}, k}(X), l \in[0, k]$ be an integer and $g d \tau \in \mathscr{B}_{\mathrm{Loc}}^{\infty}(X) \times$ $\left(\operatorname{Lip}_{\mathrm{Loc}}(X)\right)^{l}$. We define $T\left\llcorner(g d \tau) \in \mathbf{M}_{\mathrm{Loc}, k-l}(X)\right.$ by

$$
\left(T\llcorner(g d \tau))(f d \pi):=T\left(f g, \tau_{1}, \ldots, \tau_{l}, \pi_{1}, \ldots, \pi_{k-l}\right)\right.
$$

for $f d \pi \in \mathcal{D}^{k-l}(X)$, where the right-hand side of (2.3) is well-defined by Definition 2.9. $T\llcorner(g d \tau)$ is called the restriction of $T$ to $g d \tau$.

Definition 2.11. Let $T \in \mathbf{M}_{\mathrm{Loc}, k}(X)$. For a Borel set $A \subset X$, we define the restriction of $T$ to $A$ by

$$
T\left\llcorner A:=T\left\llcorner\chi_{A}\right.\right.
$$

where $\chi_{A}$ is the indicator function of $A$.

Using above notions, we define $k$-dimensional integral currents. We say $S \subset X$ is a compact $k$-rectifiable set if there exist finite compact sets $K_{1}, \ldots, K_{N}$ in $\mathbb{R}^{k}$ and $\pi_{i} \in \operatorname{Lip}\left(K_{i}, X\right)(i=1, \ldots, N)$ such that $S=\bigcup_{i=1}^{N} \pi_{i}\left(K_{i}\right)$.

Definition 2.12. A $k$-dimensional metric functional $T$ is said to be a $k$-dimensional locally integer rectifiable current if the following two conditions are satisfied:

(i) For any bounded open set $O \subset X$, we see that $\|T\|(O)<\infty$ and that for any $\epsilon>0$ there exists a compact $k$-rectifiable set $K$ such that $\|T\|(O \backslash K)<\epsilon$.

(ii) For any bounded Borel set $B \subset X$ and $\pi \in \operatorname{Lip}\left(X, \mathbb{R}^{k}\right)$, there exists $\theta \in L^{1}\left(\mathbb{R}^{k}, \mathbb{Z}\right)$ such that $\pi_{\#}(T\llcorner B)=[\theta]$.

$\mathcal{I}_{\text {Loc }, k}(X)$ denotes the set of all $k$-dimensional locally integer rectifiable currents.

Definition 2.13. $T \in \mathcal{I}_{\text {Loc }, k}(X)$ is said to be a $k$-dimensional locally integral current if $\partial T \in \mathcal{I}_{\mathrm{Loc}, k-1}(X)$ holds. $\mathbf{I}_{\mathrm{Loc}, k}(X)$ denotes the set of all $k$-dimensional locally integral currents.

Finally, we introduce a notion of convergence in $\mathbf{I}_{\mathrm{Loc}, k}(X)$.

Definition 2.14. Let $T_{n}, T \in \mathbf{I}_{\mathrm{Loc}, k}(X)$. We say $T_{n}$ converges to $T$ in the local flat topology if for any bounded closed set $B \subset X$ there exists $U_{n} \in \mathbf{I}_{\mathrm{Loc}, k}(X)$ and $V_{n} \in \mathbf{I}_{\mathrm{Loc}, k+1}(X)$ such that $T_{n}-T=U_{n}+\partial V_{n}$ and $\left(\left\|U_{n}\right\|+\left\|V_{n}\right\|\right)(B) \rightarrow 0(n \rightarrow \infty)$. 


\section{The pointed intrinsic flat distance}

In this section, we introduce the pointed intrinsic flat distance $d_{\mathcal{F} *}$ (Definition 3.1). It is a distance between pointed locally integral current spaces, which may have infinite mass. Note that the intrinsic flat distance $d_{\mathcal{F}}$ in [SW11] deals with integral current spaces, which have finite mass.

Let $k \in \mathbb{Z}_{\geq 1}$. For a complete separable metric space $X, x \in X$ and $T \in \mathbf{I}_{\mathrm{Loc}, k}(X)$, we call a triplet $(X, x, T)$ a $k$-dimensional pointed locally integral current space. The set of all $k$-dimensional pointed locally integral current spaces is denoted by $\mathcal{M}_{*}^{k}$.

Definition 3.1. Let $\left(X_{1}, x_{1}, T_{1}\right),\left(X_{2}, x_{2}, T_{2}\right) \in \mathcal{M}_{*}^{k}$. We define the pointed intrinsic flat distance between $\left(X_{1}, x_{1}, T_{1}\right)$ and $\left(X_{2}, x_{2}, T_{2}\right)$ by

$$
d_{\mathcal{F} *}\left(\left(X_{1}, x_{1}, T_{1}\right),\left(X_{2}, x_{2}, T_{2}\right)\right):=\min \left\{\widetilde{d_{\mathcal{F} *}}\left(\left(X_{1}, x_{1}, T_{1}\right),\left(X_{2}, x_{2}, T_{2}\right)\right), \frac{1}{2}\right\}
$$

where $\widetilde{d_{\mathcal{F} *}}\left(\left(X_{1}, x_{1}, T_{1}\right),\left(X_{2}, x_{2}, T_{2}\right)\right)$ is the infimum of $\epsilon>0$ satisfying following conditions: there exist a complete metric space $Z$ and an isometric embedding $\varphi_{i}: X_{i} \hookrightarrow Z(i=1,2)$ such that

(i) $d_{Z}\left(\varphi_{1}\left(x_{1}\right), \varphi_{2}\left(x_{2}\right)\right)<\epsilon$,

(ii) for $i=1,2$, there exist $U_{i} \in \mathbf{I}_{\mathrm{Loc}, k}(Z)$ and $V_{i} \in \mathbf{I}_{\mathrm{Loc}, k+1}(Z)$ such that $\varphi_{1 \#} T_{1}-$ $\varphi_{2 \#} T_{2}=U_{i}+\partial V_{i}$ and $\left(\left\|U_{i}\right\|+\left\|V_{i}\right\|\right)\left(\bar{B}_{1 / \epsilon}\left(\varphi_{i}\left(x_{i}\right)\right)\right)<\epsilon$.

Let us check that $d_{\mathcal{F} *}$ is a pseudodistance on $\mathcal{M}_{*}^{k}$. We recall the gluing of two metric spaces along same isometric images.

Lemma 3.2. Let $X, Z^{1}, Z^{2}$ be metric spaces and $\varphi^{i}: X \hookrightarrow Z^{i}(i=1,2)$ be an isometric embedding. Define $d:\left(Z^{1} \sqcup Z^{2}\right) \times\left(Z^{1} \sqcup Z^{2}\right) \rightarrow[0, \infty)$ by

$$
d\left(z, z^{\prime}\right):= \begin{cases}d_{Z^{1}}\left(z, z^{\prime}\right) & \left(z, z^{\prime} \in Z^{1}\right), \\ d_{Z^{2}}\left(z, z^{\prime}\right) & \left(z, z^{\prime} \in Z^{2}\right), \\ \inf _{x \in X}\left(d_{Z^{1}}\left(z, \varphi^{1}(x)\right)+d_{Z^{2}}\left(\varphi^{2}(x), z^{\prime}\right)\right) & \left(z \in Z^{1}, z^{\prime} \in Z^{2}\right), \\ \inf _{x \in X}\left(d_{Z^{2}}\left(z, \varphi^{2}(x)\right)+d_{Z^{1}}\left(\varphi^{1}(x), z^{\prime}\right)\right) & \left(z \in Z^{2}, z^{\prime} \in Z^{1}\right),\end{cases}
$$

then $d$ is a pseudodistance on $Z^{1} \sqcup Z^{2}$. Moreover, let $\left(Z^{1} \sqcup Z^{2}\right) / d$ be the quotient metric space, that is, let $\left(Z^{1} \sqcup Z^{2}\right) / d$ be the quotient space with respect to the equivalence relation defined by

$$
z \sim z^{\prime} \Leftrightarrow d\left(z, z^{\prime}\right)=0\left(z, z^{\prime} \in Z^{1} \sqcup Z^{2}\right) .
$$

Then the canonical inclusion $\iota^{i}: Z^{i} \hookrightarrow\left(Z^{1} \sqcup Z^{2}\right) / d(i=1,2)$ is an isometric embedding. In the following, $Z^{1} \sqcup_{X} Z^{2}$ denotes $\left(Z^{1} \sqcup Z^{2}\right) / d$.

Proof. It is enough to check that $d$ satisfies the triangle inequality;

$$
d\left(z, z^{\prime \prime}\right) \leq d\left(z, z^{\prime}\right)+d\left(z^{\prime}, z^{\prime \prime}\right) \text { for any } z, z^{\prime}, z^{\prime \prime} \in Z^{1} \sqcup Z^{2} .
$$

If $z \in Z^{1}, z^{\prime}, z^{\prime \prime} \in Z^{2}$, (3.1) follows from that for all $x \in X$

$$
d\left(z, z^{\prime \prime}\right) \leq d_{Z^{1}}\left(z, \varphi^{1}(x)\right)+d_{Z^{2}}\left(\varphi^{2}(x), z^{\prime \prime}\right) \leq d_{Z^{1}}\left(z, \varphi^{1}(x)\right)+d_{Z^{2}}\left(\varphi^{2}(x), z^{\prime}\right)+d_{Z^{2}}\left(z^{\prime}, z^{\prime \prime}\right)
$$


holds. If $z, z^{\prime \prime} \in Z^{1}, z^{\prime} \in Z^{2}$, (3.1) follows from that for all $x, x^{\prime} \in X$

$$
\begin{aligned}
d\left(z, z^{\prime \prime}\right) & \leq d_{Z^{1}}\left(z, \varphi^{1}(x)\right)+d_{Z^{1}}\left(\varphi^{1}(x), \varphi^{1}\left(x^{\prime}\right)\right)+d_{Z^{1}}\left(\varphi^{1}\left(x^{\prime}\right), z^{\prime \prime}\right) \\
& =d_{Z^{1}}\left(z, \varphi^{1}(x)\right)+d_{Z^{2}}\left(\varphi^{2}(x), \varphi^{2}\left(x^{\prime}\right)\right)+d_{Z^{1}}\left(\varphi^{1}\left(x^{\prime}\right), z^{\prime \prime}\right) \\
& =d_{Z^{1}}\left(z, \varphi^{1}(x)\right)+d_{Z^{2}}\left(\varphi^{2}(x), z^{\prime}\right)+d_{Z^{2}}\left(z^{\prime}, \varphi^{2}\left(x^{\prime}\right)\right)+d_{Z^{1}}\left(\varphi^{1}\left(x^{\prime}\right), z^{\prime \prime}\right)
\end{aligned}
$$

holds. Similarly, we can prove (3.1) in the remaining cases.

Proposition 3.3. $d_{\mathcal{F} *}$ is a pseudodistance on $\mathcal{M}_{*}^{k}$.

Proof. We use a simplified notation $d_{\mathcal{F} *}\left(X_{i}, X_{j}\right)$ (resp. $\left.\widetilde{d_{\mathcal{F} *}}\left(X_{i}, X_{j}\right)\right)$ instead of $d_{\mathcal{F} *}\left(\left(X_{i}\right.\right.$, $\left.\left.x_{i}, T_{i}\right),\left(X_{j}, x_{j}, T_{j}\right)\right)$ (resp. $\left.\widetilde{d_{\mathcal{F} *}}\left(\left(X_{i}, x_{i}, T_{i}\right),\left(X_{j}, x_{j}, T_{j}\right)\right)\right)$. It is enough to check that $d_{\mathcal{F} *}$ satisfies the triangle inequality. For $\left(X_{i}, x_{i}, T_{i}\right) \in \mathcal{M}_{*}^{k}(i=1,2,3)$, we have to show that

$$
d_{\mathcal{F} *}\left(X_{1}, X_{3}\right) \leq d_{\mathcal{F} *}\left(X_{1}, X_{2}\right)+d_{\mathcal{F} *}\left(X_{2}, X_{3}\right)
$$

holds. Without loss of generality, we can assume that $\widetilde{d_{\mathcal{F} *}}\left(X_{1}, X_{2}\right)<1 / 2$ and $\widetilde{d_{\mathcal{F} *}}\left(X_{2}, X_{3}\right)$ $<1 / 2$. For any sufficiently small $\delta>0$ with $\widehat{d_{\mathcal{F} *}}\left(X_{1}, X_{2}\right)+\delta<1 / 2$ and $\widetilde{d_{\mathcal{F} *}}\left(X_{2}, X_{3}\right)+\delta<$ $1 / 2$, there exist $\epsilon_{1}<\widetilde{d_{\mathcal{F} *}}\left(X_{1}, X_{2}\right)+\delta$ and $\epsilon_{2}<\widetilde{d_{\mathcal{F} *}}\left(X_{2}, X_{3}\right)+\delta$ such that the following holds:

(I) there exist a complete metric space $Z^{1}$ and an isometric embedding $\varphi_{i}^{1}: X_{i} \hookrightarrow Z^{1}(i=$ $1,2)$ such that

(I-i) $d_{Z^{1}}\left(\varphi_{1}^{1}\left(x_{1}\right), \varphi_{2}^{1}\left(x_{2}\right)\right)<\epsilon_{1}$,

(I-ii) for $i=1,2$, there exist $U_{i}^{1} \in \mathbf{I}_{\mathrm{Loc}, k}\left(Z^{1}\right)$ and $V_{i}^{1} \in \mathbf{I}_{\mathrm{Loc}, k+1}\left(Z^{1}\right)$ such that $\varphi_{1 \#}^{1} T_{1}-$ $\varphi_{2 \#}^{1} T_{2}=U_{i}^{1}+\partial V_{i}^{1}$ and $\left(\left\|U_{i}^{1}\right\|+\left\|V_{i}^{1}\right\|\right)\left(\bar{B}_{1 / \epsilon_{1}}\left(\varphi_{i}^{1}\left(x_{i}\right)\right)\right)<\epsilon_{1}$.

(II) there exist a complete metric space $Z^{2}$ and an isometric embedding $\varphi_{i}^{2}: X_{i} \hookrightarrow Z^{2}(i=$ $2,3)$ such that

(II-i) $d_{Z^{2}}\left(\varphi_{2}^{2}\left(x_{2}\right), \varphi_{3}^{2}\left(x_{3}\right)\right)<\epsilon_{2}$,

(II-ii) for $i=2,3$, there exist $U_{i}^{2} \in \mathbf{I}_{\mathrm{Loc}, k}\left(Z^{2}\right)$ and $V_{i}^{2} \in \mathbf{I}_{\mathrm{Loc}, k+1}\left(Z^{2}\right)$ such that $\varphi_{2 \#}^{2} T_{2}-$ $\varphi_{3 \#}^{2} T_{3}=U_{i}^{2}+\partial V_{i}^{2}$ and $\left(\left\|U_{i}^{2}\right\|+\left\|V_{i}^{2}\right\|\right)\left(\bar{B}_{1 / \epsilon_{2}}\left(\varphi_{i}^{2}\left(x_{i}\right)\right)\right)<\epsilon_{2}$.

Now, we apply Lemma 3.2 to $\varphi_{2}^{1}: X_{2} \hookrightarrow Z^{1}$ and $\varphi_{2}^{2}: X_{2} \hookrightarrow Z^{2}$. Let $Z:=Z^{1} \sqcup_{X_{2}} Z^{2}$ and $\iota^{i}: Z^{i} \hookrightarrow Z(i=1,2)$ be the canonical inclusion, then we define an isometric embedding $\varphi_{i}: X_{i} \hookrightarrow Z(i=1,2,3)$ by

$$
\begin{aligned}
& \varphi_{1}:=\iota^{1} \circ \varphi_{1}^{1}, \\
& \varphi_{2}:=\iota^{1} \circ \varphi_{2}^{1}\left(=\iota^{2} \circ \varphi_{2}^{2}\right), \\
& \varphi_{3}:=\iota^{2} \circ \varphi_{3}^{2} .
\end{aligned}
$$

Then,

$$
\begin{aligned}
d_{Z}\left(\varphi_{1}\left(x_{1}\right), \varphi_{3}\left(x_{3}\right)\right) & \leq d_{Z}\left(\varphi_{1}\left(x_{1}\right), \varphi_{2}\left(x_{2}\right)\right)+d_{Z}\left(\varphi_{2}\left(x_{2}\right), \varphi_{3}\left(x_{3}\right)\right) \\
& =d_{Z^{1}}\left(\varphi_{1}^{1}\left(x_{1}\right), \varphi_{2}^{1}\left(x_{2}\right)\right)+d_{Z^{2}}\left(\varphi_{2}^{2}\left(x_{2}\right), \varphi_{3}^{2}\left(x_{3}\right)\right) \\
& <\epsilon_{1}+\epsilon_{2}
\end{aligned}
$$


and for $i=1,2$,

$$
\begin{aligned}
\varphi_{1 \#} T_{1}-\varphi_{3 \#} T_{3} & =\left(\varphi_{1 \#} T_{1}-\varphi_{2 \#} T_{2}\right)+\left(\varphi_{2 \#} T_{2}-\varphi_{3 \#} T_{3}\right) \\
& =\iota_{\#}^{1}\left(\varphi_{1 \#}^{1} T_{1}-\varphi_{2 \#}^{1} T_{2}\right)+\iota_{\#}^{2}\left(\varphi_{2 \#}^{2} T_{2}-\varphi_{3 \#}^{2} T_{3}\right) \\
& =\iota_{\#}^{1}\left(U_{i}^{1}+\partial V_{i}^{1}\right)+\iota_{\#}^{2}\left(U_{i+1}^{2}+\partial V_{i+1}^{2}\right) \\
& =\left(\iota_{\#}^{1} U_{i}^{1}+\iota_{\#}^{2} U_{i+1}^{2}\right)+\partial\left(\iota_{\#}^{1} V_{i}^{1}+\iota_{\#}^{2} V_{i+1}^{2}\right)
\end{aligned}
$$

holds. Moreover, we have

$$
\begin{aligned}
& \left(\left\|\iota_{\#}^{1} U_{1}^{1}+\iota_{\#}^{2} U_{2}^{2}\right\|+\left\|\iota_{\#}^{1} V_{1}^{1}+\iota_{\#}^{2} V_{2}^{2}\right\|\right)\left(\bar{B}_{1 /\left(\epsilon_{1}+\epsilon_{2}\right)}\left(\varphi_{1}\left(x_{1}\right)\right)\right) \\
& \leq\left(\left\|\iota_{\#}^{1} U_{1}^{1}\right\|+\left\|\iota_{\#}^{1} V_{1}^{1}\right\|\right)\left(\bar{B}_{1 /\left(\epsilon_{1}+\epsilon_{2}\right)}\left(\varphi_{1}\left(x_{1}\right)\right)\right)+\left(\left\|\iota_{\#}^{2} U_{2}^{2}\right\|+\left\|\iota_{\#}^{2} V_{2}^{2}\right\|\right)\left(\bar{B}_{1 /\left(\epsilon_{1}+\epsilon_{2}\right)}\left(\varphi_{1}\left(x_{1}\right)\right)\right) \\
& \leq\left(\left\|\iota_{\#}^{1} U_{1}^{1}\right\|+\left\|\iota_{\#}^{1} V_{1}^{1}\right\|\right)\left(\bar{B}_{1 / \epsilon_{1}}\left(\varphi_{1}\left(x_{1}\right)\right)\right)+\left(\left\|\iota_{\#}^{2} U_{2}^{2}\right\|+\left\|\iota_{\#}^{2} V_{2}^{2}\right\|\right)\left(\bar{B}_{1 / \epsilon_{2}}\left(\varphi_{2}\left(x_{2}\right)\right)\right) \\
& \leq\left(\left\|U_{1}^{1}\right\|+\left\|V_{1}^{1}\right\|\right)\left(\bar{B}_{1 / \epsilon_{1}}\left(\varphi_{1}^{1}\left(x_{1}\right)\right)\right)+\left(\left\|U_{2}^{2}\right\|+\left\|V_{2}^{2}\right\|\right)\left(\bar{B}_{1 / \epsilon_{2}}\left(\varphi_{2}^{2}\left(x_{2}\right)\right)\right) \\
& <\epsilon_{1}+\epsilon_{2},
\end{aligned}
$$

where we used the fact that $\bar{B}_{1 /\left(\epsilon_{1}+\epsilon_{2}\right)}\left(\varphi_{1}\left(x_{1}\right)\right) \subset \bar{B}_{1 / \epsilon_{2}}\left(\varphi_{2}\left(x_{2}\right)\right)$, which follows from inequalities; $d_{Z}\left(\varphi_{1}\left(x_{1}\right), \varphi_{2}\left(x_{2}\right)\right)<\epsilon_{1}, \epsilon_{1}<1 / 2$ and $\epsilon_{2}<1 / 2$. Similarly, we have

$$
\left(\left\|\iota_{\#}^{1} U_{2}^{1}+\iota_{\#}^{2} U_{3}^{2}\right\|+\left\|\iota_{\#}^{1} V_{2}^{1}+\iota_{\#}^{2} V_{3}^{2}\right\|\right)\left(\bar{B}_{1 /\left(\epsilon_{1}+\epsilon_{2}\right)}\left(\varphi_{3}\left(x_{3}\right)\right)\right)<\epsilon_{1}+\epsilon_{2} .
$$

From the above, we have

$$
d_{\mathcal{F} *}\left(X_{1}, X_{3}\right) \leq \epsilon_{1}+\epsilon_{2}<d_{\mathcal{F} *}\left(X_{1}, X_{2}\right)+d_{\mathcal{F} *}\left(X_{2}, X_{3}\right)+2 \delta .
$$

Letting $\delta \rightarrow 0$ completes the proof.

Let us check that the convergence in Theorem 1.1 in [LW11] coincides with that by the pointed intrinsic flat distance. First, the following proposition shows that the convergence in [LW11] implies that of $d_{\mathcal{F} *}$.

Proposition 3.4. Let $\left\{\left(X_{n}, x_{n}, T_{n}\right)\right\}_{n} \subset \mathcal{M}_{*}^{k}$ be a sequence of $k$-dimensional pointed locally integral current spaces. Assume that there exist $(Z, z, T) \in \mathcal{M}_{*}^{k}$ and an isometric embedding $\varphi_{n}: X_{n} \hookrightarrow Z$ such that $\varphi_{n}\left(x_{n}\right) \rightarrow z(n \rightarrow \infty)$ and $\varphi_{n \#} T_{n}$ converges to $T$ in the local flat topology. Then $d_{\mathcal{F} *}\left(\left(X_{n}, x_{n}, T_{n}\right),(Z, z, T)\right) \rightarrow 0(n \rightarrow \infty)$.

Proof. Fix an arbitrary $\epsilon \in(0,1 / 2)$. Then for sufficiently large $n, d_{Z}\left(\varphi_{n}\left(x_{n}\right), z\right)<\epsilon$ holds, and there exist $U_{n} \in \mathbf{I}_{\mathrm{Loc}, k}(Z)$ and $V_{n} \in \mathbf{I}_{\mathrm{Loc}, k+1}(Z)$ such that $\varphi_{n \#} T_{n}-T=U_{n}+\partial V_{n}$ and $\left(\left\|U_{n}\right\|+\left\|V_{n}\right\|\right)\left(\bar{B}_{1+1 / \epsilon}(z)\right)<\epsilon$ (recall Definition 2.14). Since $\bar{B}_{1 / \epsilon}\left(\varphi_{n}\left(x_{n}\right)\right) \subset \bar{B}_{1+1 / \epsilon}(z)$, the conclusion follows.

Next, let us check that the converse of Proposition 3.4 is also true. We use the following lemma in order to prove that (Proposition 3.6).

Lemma 3.5. Let $Z, Z^{i}(i=1,2, \ldots)$ be complete metric spaces and $\varphi^{i}: Z \hookrightarrow Z^{i}(i=$ $1,2, \ldots)$ be an isometric embedding. We define $d:\left(\bigsqcup_{i=1}^{\infty} Z^{i}\right) \times\left(\bigsqcup_{i=1}^{\infty} Z^{i}\right) \rightarrow[0, \infty)$ by

$$
d\left(z, z^{\prime}\right):= \begin{cases}d_{Z^{i}}\left(z, z^{\prime}\right) & (i=j), \\ \inf _{\bar{z} \in Z}\left(d_{Z^{i}}\left(z, \varphi^{i}(\bar{z})\right)+d_{Z^{j}}\left(\varphi^{j}(\bar{z}), z^{\prime}\right)\right) & (i \neq j),\end{cases}
$$

where $z \in Z^{i}, z^{\prime} \in Z^{j}$. Then $d$ is a pseudodistance on $\bigsqcup_{i=1}^{\infty} Z^{i}$. Moreover, $\left(\bigsqcup_{i=1}^{\infty} Z^{i}\right) / d$ is a complete metric space and the canonical inclusion $\iota^{i}: Z^{i} \hookrightarrow\left(\bigsqcup_{i=1}^{\infty} Z^{i}\right) / d$ is an isometric embedding. In the following, we will use a simplified notation $\left(\bigsqcup_{i=1}^{\infty} Z^{i}\right) / Z$ instead of $\left(\bigsqcup_{i=1}^{\infty} Z^{i}\right) / d$. 
Proof. Let us check that $d$ satisfies the triangle inequality. For $z \in Z^{i}, z^{\prime} \in Z^{j}$ and $z^{\prime \prime} \in Z^{k}$, we have to show

$$
d\left(z, z^{\prime \prime}\right) \leq d\left(z, z^{\prime}\right)+d\left(z^{\prime}, z^{\prime \prime}\right)
$$

If $i, j$ and $k$ are different each other, then (3.2) holds because we have

$$
\begin{aligned}
d\left(z, z^{\prime \prime}\right) & \leq d_{Z^{i}}\left(z, \varphi^{i}(\bar{z})\right)+d_{Z^{k}}\left(\varphi^{k}(\bar{z}), z^{\prime \prime}\right) \\
& \leq d_{Z^{i}}\left(z, \varphi^{i}(\bar{z})\right)+d_{Z^{k}}\left(\varphi^{k}(\bar{z}), \varphi^{k}(\tilde{z})\right)+d_{Z^{k}}\left(\varphi^{k}(\tilde{z}), z^{\prime \prime}\right) \\
& =d_{Z^{i}}\left(z, \varphi^{i}(\bar{z})\right)+d_{Z^{j}}\left(\varphi^{j}(\bar{z}), \varphi^{j}(\tilde{z})\right)+d_{Z^{k}}\left(\varphi^{k}(\tilde{z}), z^{\prime \prime}\right) \\
& \leq d_{Z^{i}}\left(z, \varphi^{i}(\bar{z})\right)+d_{Z^{j}}\left(\varphi^{j}(\bar{z}), z^{\prime}\right)+d_{Z^{j}}\left(z^{\prime}, \varphi^{j}(\tilde{z})\right)+d_{Z^{k}}\left(\varphi^{k}(\tilde{z}), z^{\prime \prime}\right)
\end{aligned}
$$

for any $\bar{z}, \tilde{z} \in Z$. Otherwise, (3.2) holds by Lemma 3.2.

Next, we show that $\left(\bigsqcup_{i=1}^{\infty} Z^{i}\right) / d$ is a complete metric space. Let $\left\{z_{n}\right\}_{n} \subset\left(\bigsqcup_{i=1}^{\infty} Z^{i}\right) / d$ be a Cauchy sequence. If $\#\left\{z_{n} ; z_{n} \in Z^{i}\right\}=\infty$ for some $i$, we can choose a subsequence $\left\{z_{n(j)}\right\}_{j}$ such that $z_{n(j)} \in Z^{i}$ holds for all $j$. Since $\left\{z_{n(j)}\right\}_{j}$ converges to some $z \in Z^{i},\left\{z_{n}\right\}_{n}$ is a convergent sequence in $\left(\bigsqcup_{i=1}^{\infty} Z^{i}\right) / d$. If $\#\left\{z_{n} ; z_{n} \in Z^{i}\right\}<\infty$ for any $i$, after taking a subsequence, there exists a strictly increasing sequence $\{i(n)\}_{n}$ such that $z_{n} \in Z^{i(n)}$. Since $\left\{z_{n}\right\}_{n}$ is a Cauchy sequence, we have

$$
d_{Z^{i(n)}}\left(z_{n}, \varphi^{i(n)}(Z)\right) \rightarrow 0(n \rightarrow \infty) .
$$

Let $\bar{z}_{n} \in Z$ with $d_{Z^{i(n)}}\left(z_{n}, \varphi^{i(n)}\left(\bar{z}_{n}\right)\right)<d_{Z^{i(n)}}\left(z_{n}, \varphi^{i(n)}(Z)\right)+1 / 2^{n}$, then

$$
\begin{aligned}
d_{Z}\left(\bar{z}_{n}, \bar{z}_{m}\right) & =d\left(\varphi^{i(n)}\left(\bar{z}_{n}\right), \varphi^{i(m)}\left(\bar{z}_{m}\right)\right) \\
& \leq d\left(\varphi^{i(n)}\left(\bar{z}_{n}\right), z_{n}\right)+d\left(z_{n}, z_{m}\right)+d\left(z_{m}, \varphi^{i(m)}\left(\bar{z}_{m}\right)\right) \\
& =d_{Z^{i(n)}}\left(\varphi^{i(n)}\left(\bar{z}_{n}\right), z_{n}\right)+d\left(z_{n}, z_{m}\right)+d_{Z^{i(m)}}\left(z_{m}, \varphi^{i(m)}\left(\bar{z}_{m}\right)\right) \\
& \rightarrow 0(n, m \rightarrow \infty) .
\end{aligned}
$$

Hence $\left\{\bar{z}_{n}\right\}_{n} \subset Z$ is a Cauchy sequence and converges to some $\bar{z} \in Z$. Here,

$$
\begin{aligned}
d\left(\varphi^{i(n)}(\bar{z}), z_{n}\right) & =d_{Z^{i(n)}}\left(\varphi^{i(n)}(\bar{z}), z_{n}\right) \\
& \leq d_{Z^{i(n)}}\left(\varphi^{i(n)}(\bar{z}), \varphi^{i(n)}\left(\bar{z}_{n}\right)\right)+d_{Z^{i(n)}}\left(\varphi^{i(n)}\left(\bar{z}_{n}\right), z_{n}\right) \\
& =d_{Z}\left(\bar{z}, \bar{z}_{n}\right)+d_{Z^{i(n)}}\left(\varphi^{i(n)}\left(\bar{z}_{n}\right), z_{n}\right) \\
& \rightarrow 0(n \rightarrow \infty)
\end{aligned}
$$

holds. Since $d\left(\varphi^{i(n)}(\bar{z}), \varphi^{i(m)}(\bar{z})\right)=0$ for any $n, m \in \mathbb{N}$, the conclusion follows.

Now we show the converse of Proposition 3.4.

Proposition 3.6. Assume that $\left(X_{n}, x_{n}, T_{n}\right),(Z, z, T) \in \mathcal{M}_{*}^{k}$ satisfy $d_{\mathcal{F} *}\left(\left(X_{n}, x_{n}, T_{n}\right),(Z\right.$, $z, T)) \rightarrow 0(n \rightarrow \infty)$. Then there exist $\left(Z^{\prime}, z^{\prime}, T^{\prime}\right) \in \mathcal{M}_{*}^{k}$ and an isometric embedding $\varphi_{n}: X_{n} \hookrightarrow Z^{\prime}$ such that $\varphi_{n}\left(x_{n}\right) \rightarrow z^{\prime}(n \rightarrow \infty)$ and that $\varphi_{n \#} T_{n}$ converges to $T^{\prime}$ in the local flat topology. Moreover, we can take $\left(Z^{\prime}, z^{\prime}, T^{\prime}\right)$ in such a way that there exists an isometric embedding $\varphi: Z \hookrightarrow Z^{\prime}$ satisfying $\varphi(z)=z^{\prime}$ and $\varphi_{\#} T=T^{\prime}$.

Proof. Let $\epsilon_{n}:=\widetilde{d_{\mathcal{F} *}}\left(\left(X_{n}, x_{n}, T_{n}\right),(Z, z, T)\right)$. By Definition 3.1, there exists $\delta_{n} \in\left[\epsilon_{n}, \epsilon_{n}+\right.$ $1 / 2^{n}$ ) (if $\epsilon_{n}=0, \delta_{n} \in\left(0,1 / 2^{n}\right)$ ) satisfying following conditions:

There exist a complete metric space $Z^{n}$ and isometric embeddings $\varphi^{n}: X_{n} \hookrightarrow Z^{n}, \psi^{n}$ : $Z \hookrightarrow Z^{n}$ such that 
(i) $d_{Z^{n}}\left(\varphi^{n}\left(x_{n}\right), \psi^{n}(z)\right)<\delta_{n}$,

(ii) there exist $U_{n}, \tilde{U}_{n} \in \mathbf{I}_{\mathrm{Loc}, k}\left(Z^{n}\right), V_{n}, \tilde{V}_{n} \in \mathbf{I}_{\mathrm{Loc}, k+1}\left(Z^{n}\right)$ such that $\varphi_{\#}^{n} T_{n}-\psi_{\#}^{n} T=$ $U_{n}+\partial V_{n}=\tilde{U}_{n}+\partial \tilde{V}_{n}$, that $\left(\left\|U_{n}\right\|+\left\|V_{n}\right\|\right)\left(\bar{B}_{1 / \delta_{n}}\left(\varphi^{n}\left(x_{n}\right)\right)\right)<\delta_{n}$ and that $\left(\left\|\tilde{U}_{n}\right\|+\right.$ $\left.\left\|\tilde{V}_{n}\right\|\right)\left(\bar{B}_{1 / \delta_{n}}\left(\psi^{n}(z)\right)\right)<\delta_{n}$.

Now we apply Lemma 3.5 to $\psi^{n}: Z \hookrightarrow Z^{n}$. Let $Z^{\prime}:=\left(\bigsqcup_{n=1}^{\infty} Z^{n}\right) / Z$ and $\phi_{n}: Z^{n} \hookrightarrow$ $Z^{\prime}, \varphi: Z \hookrightarrow Z^{\prime}$ be the canonical isometric embeddings. If we take $z^{\prime}:=\varphi(z), T^{\prime}:=\varphi_{\#} T$ and $\varphi_{n}:=\phi_{n} \circ \varphi^{n}$, the conclusion follows. In fact,

$$
d_{Z^{\prime}}\left(z^{\prime}, \varphi_{n}\left(x_{n}\right)\right)=d_{Z^{n}}\left(\psi^{n}(z), \varphi^{n}\left(x_{n}\right)\right)<\delta_{n}
$$

implies the convergence of the reference point. Moreover, since

$$
\varphi_{n \#} T_{n}-\varphi_{\#} T=\phi_{n \#}\left(\varphi_{\#}^{n} T_{n}-\psi_{\#}^{n} T\right)=\phi_{n \#}\left(\tilde{U}_{n}+\partial \tilde{V}_{n}\right)=\left(\phi_{n \#} \tilde{U}_{n}\right)+\partial\left(\phi_{n \#} \tilde{V}_{n}\right),
$$

$\varphi_{n \#} T_{n}$ converges to $T^{\prime}$ in the local flat topology because

$$
\begin{aligned}
\left(\left\|\phi_{n \#} \tilde{U}_{n}\right\|+\left\|\phi_{n \#} \tilde{V}_{n}\right\|\right)(B) & \leq\left(\left\|\phi_{n \#} \tilde{U}_{n}\right\|+\left\|\phi_{n \#} \tilde{V}_{n}\right\|\right)\left(\bar{B}_{1 / \delta_{n}}\left(z^{\prime}\right)\right) \\
& \leq\left(\left\|\tilde{U}_{n}\right\|+\left\|\tilde{V}_{n}\right\|\right)\left(\bar{B}_{1 / \delta_{n}}\left(\psi_{n}(z)\right)\right) \\
& <\delta_{n}
\end{aligned}
$$

where $B \subset Z^{\prime}$ is an arbitrary bounded closed set and $n$ is a sufficiently large number satisfying $B \subset \bar{B}_{1 / \delta_{n}}\left(z^{\prime}\right)$.

In the end of our note, we discuss the case of $d_{\mathcal{F} *}=0$.

Proposition 3.7. If $d_{\mathcal{F} *}\left((X, x, T),\left(X^{\prime}, x^{\prime}, T^{\prime}\right)\right)=0$, then there exists an isometry $\psi$ : $\{x\} \cup \operatorname{spt} T \rightarrow\left\{x^{\prime}\right\} \cup \operatorname{spt} T^{\prime}$ such that $\psi(x)=x^{\prime}$ and $\psi_{\#} T=T^{\prime}$.

Proof. Since $d_{\mathcal{F} *}\left((X, x, T),\left(X^{\prime}, x^{\prime}, T^{\prime}\right)\right)=0$, there exists a sequence of $\delta_{n}>0$ with $\lim _{n \rightarrow \infty} \delta_{n}=0$ and following conditions: there exist a complete metric space $Z_{n}$ and isometric embeddings $\varphi_{n}: X \hookrightarrow Z_{n}, \varphi_{n}^{\prime}: X^{\prime} \hookrightarrow Z_{n}$ such that

(i) $d_{Z_{n}}\left(\varphi_{n}(x), \varphi_{n}^{\prime}\left(x^{\prime}\right)\right)<\delta_{n}$,

(ii) there exist $U_{n}, \tilde{U}_{n} \in \mathbf{I}_{\mathrm{Loc}, k}\left(Z_{n}\right)$ and $V_{n}, \tilde{V}_{n} \in \mathbf{I}_{\mathrm{Loc}, k+1}\left(Z_{n}\right)$ such that $\varphi_{n \#} T-\varphi_{n \#}^{\prime} T^{\prime}=$ $U_{n}+\partial V_{n}=\tilde{U}_{n}+\partial \tilde{V}_{n},\left(\left\|U_{n}\right\|+\left\|V_{n}\right\|\right)\left(\bar{B}_{1 / \delta_{n}}\left(\varphi_{n}(x)\right)\right)<\delta_{n}$ and $\left(\left\|\tilde{U}_{n}\right\|+\left\|\tilde{V}_{n}\right\|\right)\left(\bar{B}_{1 / \delta_{n}}\right.$ $\left.\left(\varphi_{n}^{\prime}\left(x^{\prime}\right)\right)\right)<\delta_{n}$.

Now we apply Lemma 3.5 to $\varphi_{n}^{\prime}: X^{\prime} \hookrightarrow Z_{n}$ and let $Z:=\left(\bigsqcup_{n=1}^{\infty} Z_{n}\right) / X^{\prime}$. Then let $\iota_{n}: Z_{n} \hookrightarrow Z$ and $\psi^{\prime}: X^{\prime} \hookrightarrow Z$ be canonical isometric embeddings, and one can check $\psi_{n}(x) \rightarrow \psi^{\prime}\left(x^{\prime}\right)(n \rightarrow \infty)$ and $\psi_{n \#} T$ converges to $\psi_{\#}^{\prime} T^{\prime}$ in the local flat topology where $\psi_{n}:=\iota_{n} \circ \varphi_{n}$. Now the conclusion follows from Proposition 1.1 in [LW11].

In particular, $d_{\mathcal{F} *}$ is a distance function on the quotient space $\mathcal{M}_{*}^{k} / \sim$, where $\sim$ is an equivalence relation on $\mathcal{M}_{*}^{k}$ defined as follows: we say $(X, x, T) \sim\left(X^{\prime}, x^{\prime}, T^{\prime}\right)$ if and only if there exists an isometry $\psi:\{x\} \cup \operatorname{spt} T \rightarrow\left\{x^{\prime}\right\} \cup \operatorname{spt} T^{\prime}$ such that $\psi(x)=x^{\prime}$ and $\psi_{\#} T=T^{\prime}$. 


\section{References}

[AK00] L. Ambrosio, B. Kirchheim: Currents in metric spaces, Acta Math. 185(2000), no.1, 1-80.

[Gro81] M. Gromov: Groups of polynomial growth and expanding maps, Inst. Hautes Études Sci. Publ. Math. (1981), no.53, 53-73.

[Hon17] S. Honda: Ricci curvature and orientability, Calc. Var. Partial Differential Equations 56(2017), no.6, Art. 174, 47pp.

[LW11] U. LANG, S. WENGER: The pointed flat compactness theorem for locally integral currents, Comm. Anal. Geom. 19(2011), no.1, 159-189.

[SW11] C. Sormani, S. Wenger: The intrinsic flat distance between Riemannian manifolds and other integral current spaces, J. Differential Geom. 87(2011), no.1, 117-199.

[Wen05] S. Wenger: Isoperimetric inequalities of Euclidean type in metric spaces, Geom. Funct. Anal. 15(2005), no.2, 534-554.

[Wen07] S. Wenger: Flat convergence for integral currents in metric spaces, Calc. Var. Partial Differential Equations 28(2007), no.2, 139-160.

[Wen11] S. Wenger: Compactness for manifolds and integral currents with bounded diameter and volume, Calc. Var. Partial Differential Equations 40(2011), no.3-4, 423-448. 